\title{
Nitrogen Fixation in Wheat Provided by Klebsiella pneumoniae 342
}

\author{
A. Leonardo Iniguez, ${ }^{1}$ Yuemei Dong, ${ }^{1}$ and Eric W. Triplett $^{2}$ \\ ${ }^{1}$ University of Wisconsin-Madison, Department of Agronomy, 1575 Linden Drive, Madison 53706 U.S.A.; ${ }^{2}$ University of \\ Florida, Department of Microbiology and Cell Science, P.O. Box 110700, Gainesville 32611-0700 U.S.A.
}

Submitted 16 March 2004. Accepted 8 June 2004.

In this report, all of the criteria necessary for the demonstration of nitrogen fixation in wheat (Triticum aestivum L.), the world's most important crop, are shown upon inoculation with a nitrogen-fixing bacterium, Klebsiella pneumoniae 342 (Kp342). Kp342 relieved nitrogen (N) deficiency symptoms and increased total $N$ and $N$ concentration in the plant. Nitrogen fixation was confirmed by ${ }^{15} \mathrm{~N}$ isotope dilution in the plant tissue and in a plant product, chlorophyll. All of these observations were in contrast to uninoculated plants, plants inoculated with a nitrogen-fixing mutant of Kp342, and plants inoculated with dead Kp342 cells. Nitrogenase reductase was produced by $\mathrm{Kp342}$ in the intercellular space of the root cortex. Wild-type Kp342 and the nifH mutant colonized the interior of wheat roots in equal numbers on a fresh weight basis. The nitrogen fixation phenotype described here was specific to cv. Trenton. Inoculation of cvs. Russ or Stoa with Kp342 resulted in no relief of nitrogen deficiency symptoms.

Additional keywords: diazotrophic endophyte, endophytic colonization.

An important goal of nitrogen fixation research is the extension of this phenotype to nonleguminous plants, particularly important agronomic grasses such as wheat, rice, and maize. Despite enormous progress in understanding the development of the nitrogen-fixing symbiosis between rhizobia and legumes, the path to use that knowledge to induce nitrogen-fixing nodules on nonleguminous crops still is not clear. Similarly, transforming plant genomes with bacterial nif genes to obtain nitrogen-fixing nonlegumes remains a daunting task (Dixon et al. 1997). However, nitrogen (N)-fixing bacteria that inhabit the interior of grasses without causing any disease of symbiotic structures, called diazotrophic endophytes, may provide sufficient amounts of fixed $\mathrm{N}$ to relieve nitrogen deficiency under conditions where $\mathrm{N}$ is limiting. Definitive evidence that a particular bacterium is providing fixed $\mathrm{N}$ to the plant requires these lines of evidence: i) total plant $\mathrm{N}$ must significantly increase upon inoculation, preferably with a concomitant increase in $\mathrm{N}$ concentration in the plant; ii) nitrogen deficiency symptoms must be relieved under N-limiting conditions upon inoculation, which should include an increase in dry matter; iii) $\mathrm{N}_{2}$ fixation must be documented through the use of an ${ }^{15} \mathrm{~N}$

Corresponding author: E. W. Triplett; Telephone: 352-392-5430; Fax: 352-392-5922; E-mail: ewt@ufl.edu

Current address of Y. Dong: Johns Hopkins University, Department of Molecular Microbiology \& Immunology, 615 Wolfe St., Baltimore, MD 21205, U.S.A. approach, which can consist of isotope dilution experiments, ${ }^{15} \mathrm{~N}_{2}$ reduction assays, or ${ }^{15} \mathrm{~N}$ natural abundance assays; iv) fixed $\mathrm{N}$ must be incorporated into a plant protein or metabolite; and v) all of these effects must not be seen in uninoculated plants or in plants inoculated with an Nif- mutant of the inoculum strain. In addition, the inoculum strain must be recovered from the host plant in order to fulfill Koch's postulates. In this study, all of these criteria have been met for the first time in a grass species and are shown in wheat, the world's most important crop species.

Inoculation with several bacterial endophytes on maize in greenhouse and field experiments failed to relieve nitrogen deficiency symptoms (Riggs et al. 2001). However, one strain, Klebsiella pneumoniae 342 (Kp342), relieved nitrogen deficiency symptoms in Trenton wheat cultured in the absence of $\mathrm{N}$ fertilizer. This work shows that the nitrogen-fixing capability of Kp342 is responsible for this relief and results in fixed $\mathrm{N}$ for the plant. Kp342 is a nitrogen-fixing endophyte originally isolated from a nitrogen-efficient line of maize (Chelius and Triplett 2000). This strain has a very broad host range and is capable of colonizing the interior of many plants with fewer than 10 cells in the inoculum (Dong et al. 2003b and c).

\section{RESULTS}

After six weeks of growth in the greenhouse without nitrogen fertilizer, uninoculated plants and plants inoculated with the nifH mutant were stunted and chlorotic showing severe signs of nitrogen deficiency (Fig. 1A and B). Only wheat plants inoculated with K. pneumoniae 324 appeared taller, more robust, and greener than the controls, regardless of the medium in which they were grown (Fig. 1A and B). Two plant culture media (1:1 sand-perlite and 1:1 sand-vermiculite) were used to illustrate the reproducibility of the results. The results of dead cell inoculum treatment for all parameters measured were not statistically different from the nifH or uninoculated treatments (data not shown). Chlorophyll levels in Kp342inoculated plants were significantly higher than those levels found in control plants (Fig. 1C).

Kp342 also significantly increased the dry weight of roots and shoots compared with controls, regardless of the growth medium (Fig. 2A and B). Roots and shoots of Kp342-inoculated plants were always at least $50 \%$ larger in dry weight compared with the untreated controls. Changes in total $\mathrm{N}$ per plant with Kp342 inoculation were even more dramatic. In sand-perlite, the percent increase in total $\mathrm{N}$ for Kp342-inoculated plants grown was 244 and $498 \%$ greater for roots and shoots, respectively, compared with the nifH control (Fig. 2C and D). Compared with the uninoculated control, Kp342 accumulated 285 and $654 \%$ more total $\mathrm{N}$ in shoots and roots, re- 
spectively. In sand-vermiculite, Kp342-inoculated plants had 180 and $707 \%$ more total $\mathrm{N}$ compared with the nifH-inoculated plants in the roots and shoots, respectively. In the same growth medium, the total $\mathrm{N}$ of Kp342-inoculated plants increased 120 and $378 \%$ for roots and shoots, respectively, compared with uninoculated plants (Fig. 2C and D).

The concentration of $\mathrm{N}$ in plant tissues also increased significantly with Kp342 inoculation compared with the controls. In sand-perlite, the percent increase in total $\mathrm{N}$ concentration for Kp342-inoculated plants grown was 318 and $368 \%$ greater for roots and shoots, respectively, compared with the nifH control (Fig. 2E and G). Compared with the uninoculated control, Kp342 accumulated an N concentration 317 and 394\% higher in shoots and roots, respectively (Fig. 2E and G). In sand-vermiculite, Kp342-inoculated plants had an N concentration 161 and $381 \%$ higher than the nifH-inoculated plants in the roots and shoots, respectively (Fig. $2 \mathrm{~F}$ and $\mathrm{H}$ ). In the same growth medium, the $\mathrm{N}$ concentration of Kp342-inoculated plants increased 120 and $378 \%$, for roots and shoots, respectively, compared with uninoculated plants (Fig. 2F and $\mathrm{H}$ ).

To verify that much of the $\mathrm{N}$ in these plants was derived from the atmosphere, the plant growth media were evenly labeled with $10 \mathrm{mg}$ of 11.7 atom percent excess ${ }^{15} \mathrm{NH}_{4} \mathrm{NO}_{3}$ per kilogram of sand-vermiculite and sand-perlite mixes. The ${ }^{15} \mathrm{~N}$ concentration of Kp342-inoculated plants was significantly lower than in the controls as a result of nitrogen fixation (Fig. 3). A significant source of ${ }^{15} \mathrm{~N}$ in the plants is from the enriched ${ }^{15} \mathrm{~N}$ in the soil; therefore, the extent of the dilution of the ${ }^{15} \mathrm{~N}$ isotope can be used to calculate the amount of $\mathrm{N}$ in the plants derived from the atmosphere. This can be calculated by
$\% \mathrm{NF}=(1-A / B) \times 100$, where $\% \mathrm{NF}=$ the percent of $\mathrm{N}$ in the nitrogen-fixing system derived from the atmosphere, $A=$ percent ${ }^{15} \mathrm{~N}$ in the nitrogen-fixing system, and $B=$ percent ${ }^{15} \mathrm{~N}$ in the nonfixing system (Boddey and Chalk 1983). When the comparison is made with the nifH control, the Kp342-inoculated plants received 42 and $41 \%$ of their nitrogen from $\mathrm{N}_{2}$ for plants grown in sand-perlite and sand-vermiculite, respectively. When the comparison is made with the uninoculated control, the Kp342-inoculated plants received 49 and $37 \%$ of their nitrogen from $\mathrm{N}_{2}$ when the plants were cultured in sandperlite and sand-vermiculite, respectively.

The remaining $\mathrm{N}$ in Kp342-inoculated plants came primarily from the plant growth media because the $\mathrm{N}$ content of seed was very low, being less than $0.006 \%$ of the total $\mathrm{N}$ in the pots at the time of planting. This was calculated by determining the amount of $\mathrm{N}$ in three sets of 10 seeds taken from the same bag of seeds, the amount of $\mathrm{N}$ as ${ }^{15} \mathrm{NH}_{4} \mathrm{NO}_{3}$ added to the soil mixes, and the total amount of $\mathrm{N}$ in the soil mixes. On average, the sand-vermiculite and sand-perlite pots contained 91.6 and $74.6 \mathrm{mg}$ of $\mathrm{N}$, respectively, at the start of the experiment. This includes an average of 8.0 and $6.8 \mathrm{mg}$ of 11.7 atom percent excess ${ }^{15} \mathrm{NH}_{4} \mathrm{NO}_{3}$ in sand-vermiculite and sand-perlite, respectively. However, Kp342-inoculated plants contained 132.3 and $78.2 \mathrm{mg}$ of $\mathrm{N}$ per pot (two plants/pot) in sand-vermiculite and sand-perlite, respectively. That is, the plants contained statistically significantly more N (44 and 5\% more $\mathrm{N}$ in sand-vermiculite and sand-perlite cultured plants, respectively) than was present in the entire pot (including seed $\mathrm{N})$ at the start of the experiment. In contrast, the nifH mutantinoculated plants contained only 27.9 and $12.8 \mathrm{mg}$ of $\mathrm{N}$ per
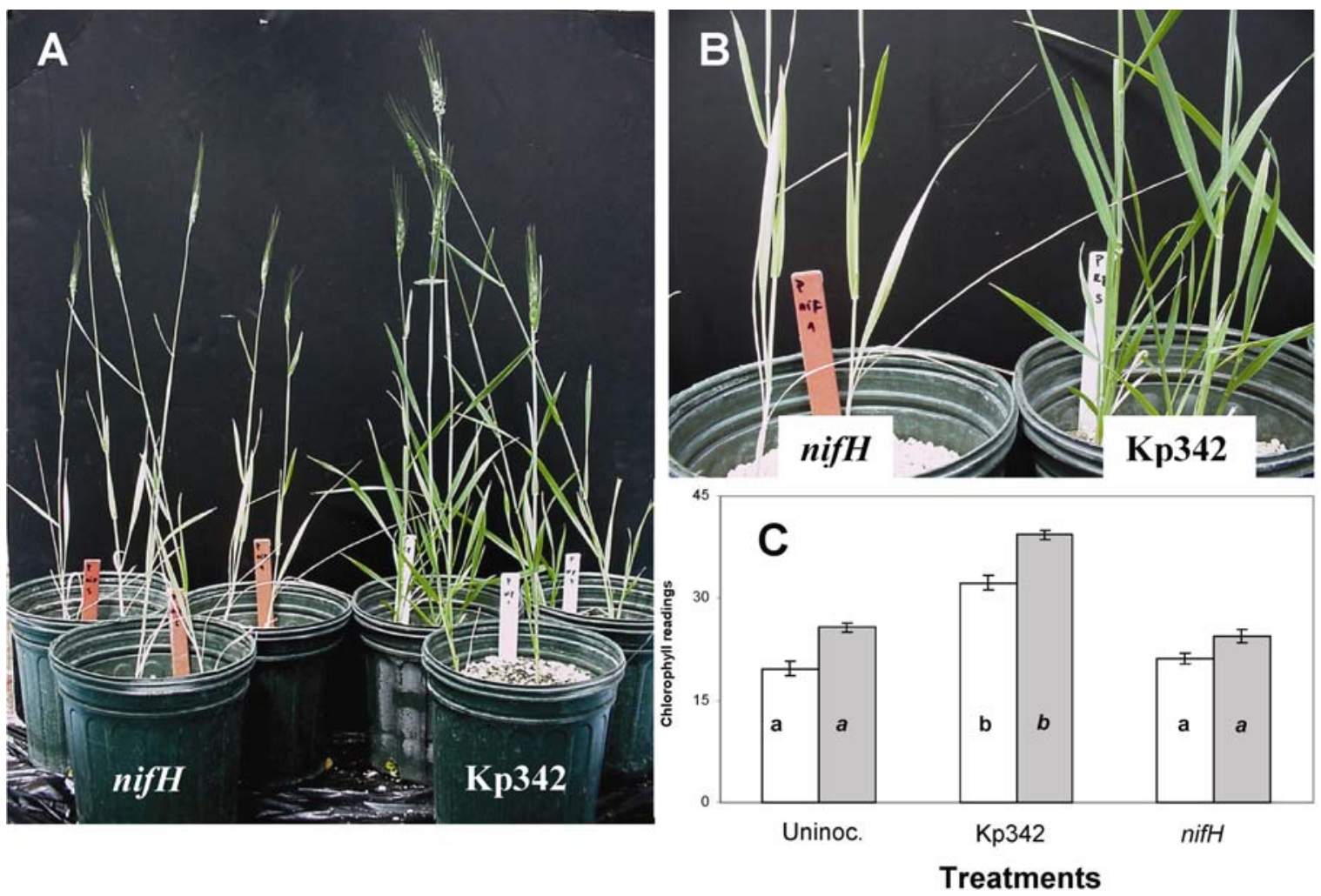

Fig. 1. Six-week-old spring wheat Triticum aestivum L. cv. Trenton inoculated with Klebsiella pneumoniae strain 342 (Kp342) and nifH mutant of Kp342 (nifH) grown in labeled (10 mg of ${ }^{15} \mathrm{NH}_{4} \mathrm{NO}_{3}$ per kilogram of soil mix) $\mathbf{A}$, sand and $\mathbf{B}$, perlite. The plants in the three pots on the left were inoculated with the nifH mutant of Kp342 while the plants in the three pots on the right were inoculated with Kp342. C, Chlorophyll readings of 6-week-old spring wheat Triticum aestivum L. cv. Trenton were obtained from Minolta SPAD 502, where the ratio of transmittance from two wavelengths (650/940 nm) creates an arbitrary unit related to chlorophyll content. Plants were inoculated with Kp342, the nifH mutant, or uninoculated (Uninoc.). Treated plants were grown in labeled (10 mg of ${ }^{15} \mathrm{NH}_{4} \mathrm{NO}_{3}$ per kilogram of soil mix) sand-perlite (open column) or sand-vermiculite (closed column). The columns represent the mean SPAD readings. The bars represent the standard error. Least significant difference statistical analysis was calculated to determine difference between treatments. These differences are represented by letters inside the columns. 
pot (two plants per pot), for the sand-vermiculite and sandperlite experiments, respectively. Thus, the nifH mutant-inoculated plants contained far less $\mathrm{N}$ than was present in the pots (including seed N) at the beginning of the experiment. The nutrient solution contained no detectable $\mathrm{N}$ throughout the experiment, with a limit of detection of $0.3 \mathrm{ppm}$. A concentration of $0.3 \mathrm{ppm} \mathrm{N}$ in the nutrient solution is insufficient to relieve the nitrogen-deficiency symptoms observed here in the uninoculated plants or plants inoculated with the nifH mutant of Kp342.

Assuming that the percent $\mathrm{N}$ in the plants derived from the atmosphere is that calculated based on the ${ }^{15} \mathrm{~N}$ abundance of nifH mutant- and Kp342-inoculated plants, the Kp342-inoculated plants were capable of mining approximately 62 and $86 \%$ of the total $\mathrm{N}$ in the growth medium for the sand-perlite and sand-vermiculite mixtures, respectively. That amount, combined with the amount of $\mathrm{N}_{2}$ fixed from the atmosphere, allowed for vigorous plant growth and relieved the nitrogendeficiency symptoms. Thus, the increased availability of $\mathrm{N}$ to the Kp342-inoculated plants permitted more root growth, allowing these plants to absorb a majority of the $\mathrm{N}$ present in the soil. In contrast, the nitrogen-limited control plants had very small roots that were able to absorb only 19 and $21 \%$ of the $\mathrm{N}$ from the growth medium for the sand-perlite and sand-vermiculite mixtures, respectively.

Fixed $\mathrm{N}$ also was incorporated into chlorophyll. Chlorophyll was extracted from the plant tissue and acidified to pheophytin. The proportion of ${ }^{15} \mathrm{~N} /{ }^{14} \mathrm{~N}$ in the four $\mathrm{N}$ atoms of pheophytin was determined by mass spectrometry. A pheophytin molecule from the nifH treatment was more than twice as likely to be fully labeled with ${ }^{15} \mathrm{~N}$ than in the Kp342 treatment,
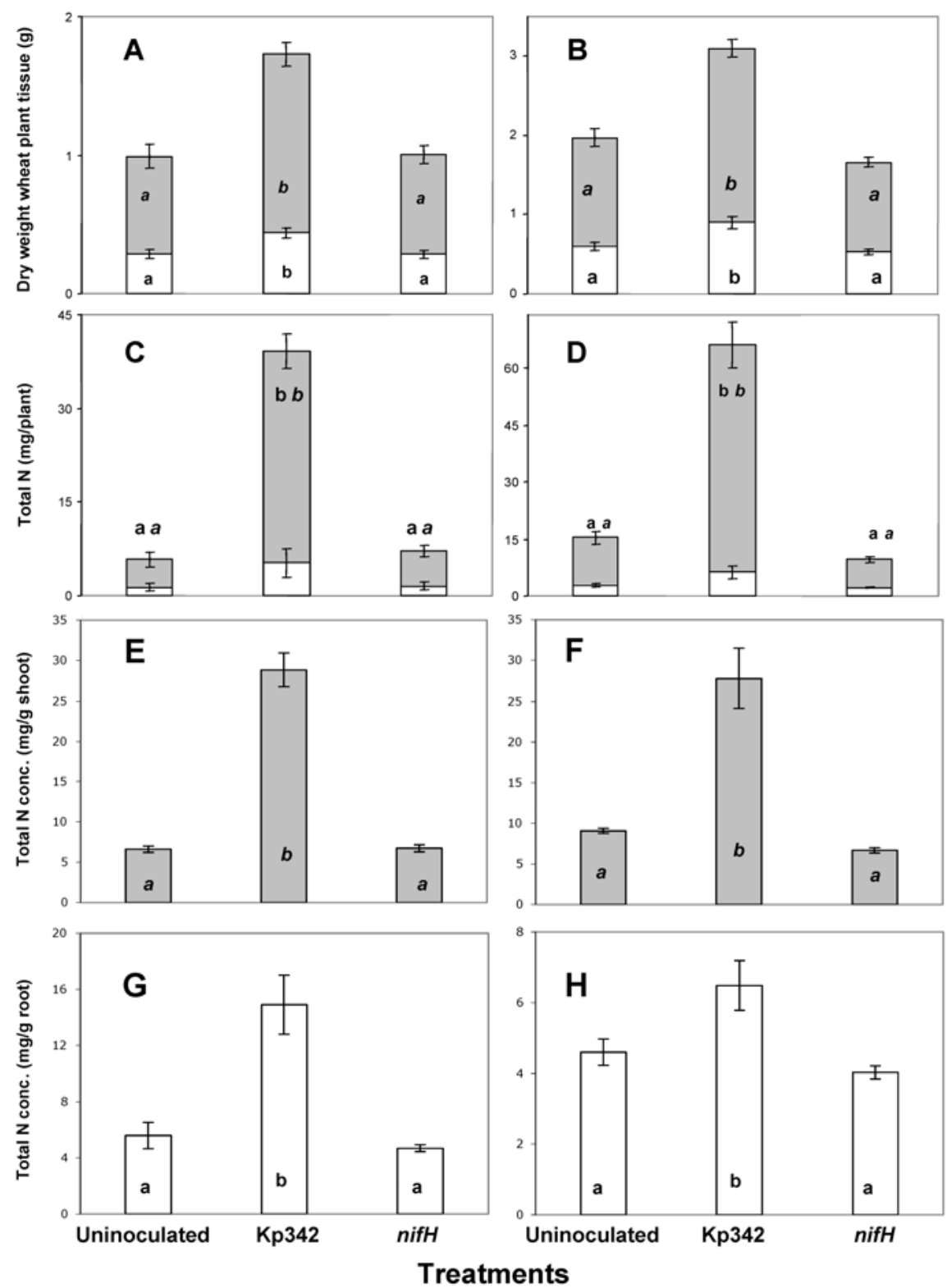

Fig. 2. Triticum aestivum L. cv. Trenton plants were inoculated with Klebsiella pneumoniae strain 342 (Kp342) and compared with uninoculated plants or inoculated with a nifH mutant. Dry roots (open columns) and shoots (closed columns) from plants grown in labeled $\left(10 \mathrm{mg}\right.$ of ${ }^{15} \mathrm{NH}_{4} \mathrm{NO}_{3}$ per kilogram of soil mix) A, C, E, and $\mathbf{G}$, sand-perlite and $\mathbf{B}, \mathbf{D}, \mathbf{F}$, and $\mathbf{H}$, sand-vermiculite were used to estimate $\mathbf{A}$ and $\mathbf{B}$, dry weights, $\mathbf{C}$ and $\mathbf{D}$, total $\mathbf{N}$ per plant, and total $\mathbf{N}$ concentration in $\mathbf{E}$ and $\mathbf{F}$, shoots and $\mathbf{G}$ and $\mathbf{H}$, roots in dried tissue, 6 weeks post inoculation. The columns represent the mean of dry weight for plants grown in $\mathbf{A}$, sand-perlite and $\mathbf{B}$, sand-vermiculite, and total nitrogen per plant grown in $\mathbf{B}$, sand-perlite and $\mathbf{C}$, sand-vermiculite. The columns also represent the mean total $\mathbf{N}$ concentration per gram of $\mathbf{E}$, dried shoot and $\mathbf{G}$, root for plants grown in $\mathbf{F}$, sand-perlite and $\mathbf{H}$, sand-vermiculite. The bars represent the standard error. Least significant difference (LSD) statistical analysis was calculated to determine difference between treatments. Letters inside the columns represents the LSD calculations (roman for roots and italics for shoots). 

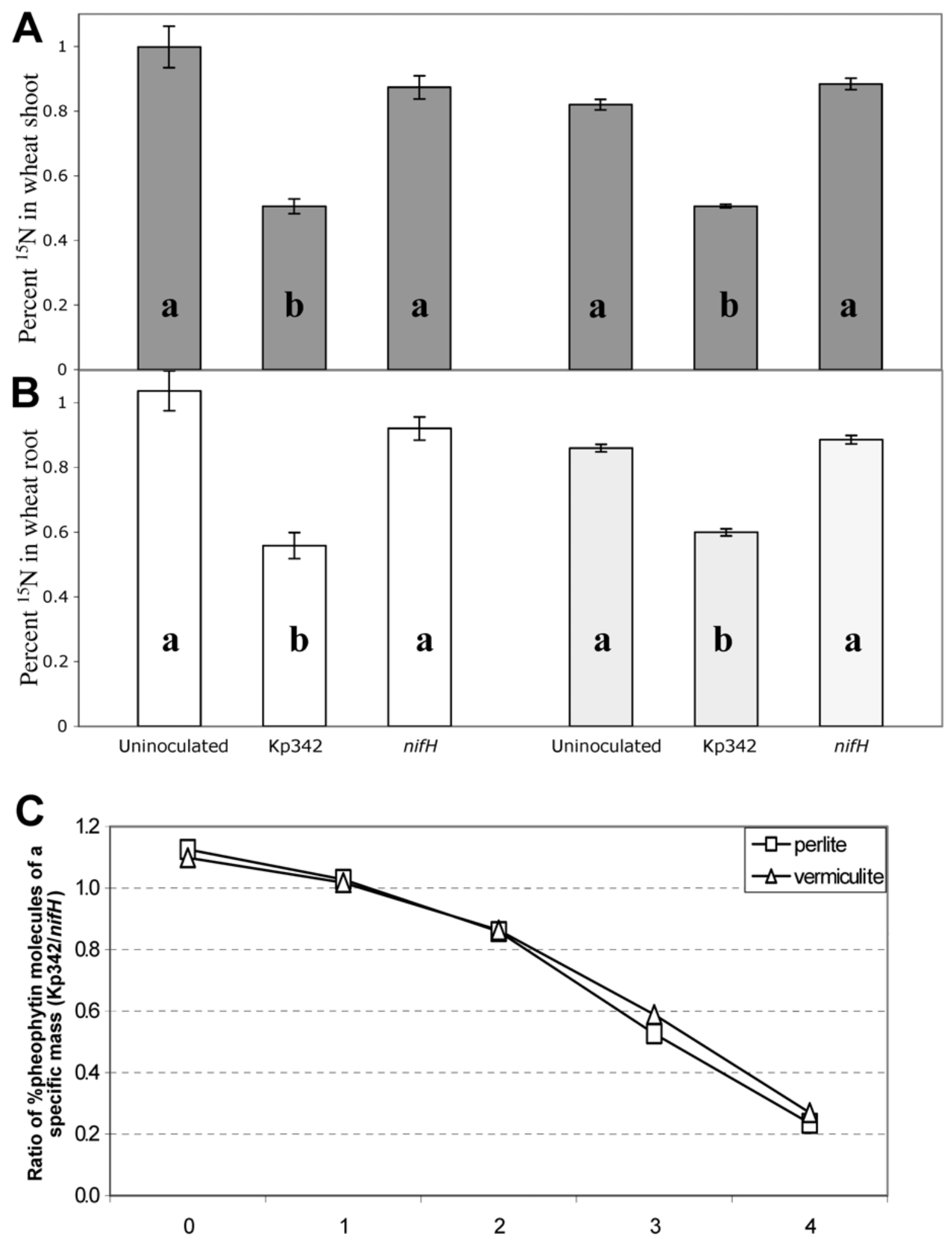

\section{No. of ${ }^{15} \mathrm{Ns}$ in pheophytin}

Fig. 3. A, Percent ${ }^{15} \mathrm{~N}$ content in 6-week-old Triticum aestivum L. cv. Trenton grown in labeled $\left({ }^{15} \mathrm{NH}_{4} \mathrm{NO}_{3}, 11.7\right.$ atom percent excess, $10 \mathrm{mg}$ per kilogram of soil mix) sand-perlite (A, solid open and $\mathbf{B}$, closed columns) and sand-vermiculite (A, dotted open and $\mathbf{B}$, closed columns). The percent ${ }^{15} \mathrm{~N}$ was analyzed from dried and ground A, shoots and B, roots. Plants were inoculated with Klebsiella pneumoniae strain 342 (Kp342), nifH mutant of Kp342 (nifH), or uninoculated (control). The columns represent the mean percent ${ }^{15} \mathrm{~N}$ in the tissue. The bars represent the standard error. Significant differences are indicated by letters within the columns. C, The phaeophytin molecule contains $4 \mathrm{~N}$ atoms. Any or all of these may be labeled with ${ }^{15} \mathrm{~N}$. This represents the ratio of the percent pheophytin molecules that contain zero to four ${ }^{15} \mathrm{~N}$ atoms in the two treatments (Kp342/nifH) versus the number of ${ }^{15} \mathrm{~N}$ atoms observed in the pheophytin molecule by mass spectrometry. 
regardless of the growth medium used for plant culture. Similarly, a significantly higher proportion of pheophytin molecules were labeled with two or three ${ }^{15} \mathrm{~N}$ atoms in the nifH treatment compared with the Kp342 treatment. Thus, just as nitrogen fixation in Kp342-inoculated plants diluted the ${ }^{15} \mathrm{~N}$ label in total plant tissue, this dilution also was observed di- rectly in a plant product, chlorophyll. The mean mass of pheophytin was $872.454( \pm 0.041), 872.234( \pm 0.0036), 872.398$ $( \pm 0.027)$, and $872.238( \pm 0.0031)$ for the nifH and Kp342 treatments in sand-perlite, and the nifH and Kp342 treatments in sand-vermiculite, respectively. The mass of pheophytin with all four $\mathrm{N}$ atoms as ${ }^{14} \mathrm{~N}$ is 871.6 . The decline in average pheo-
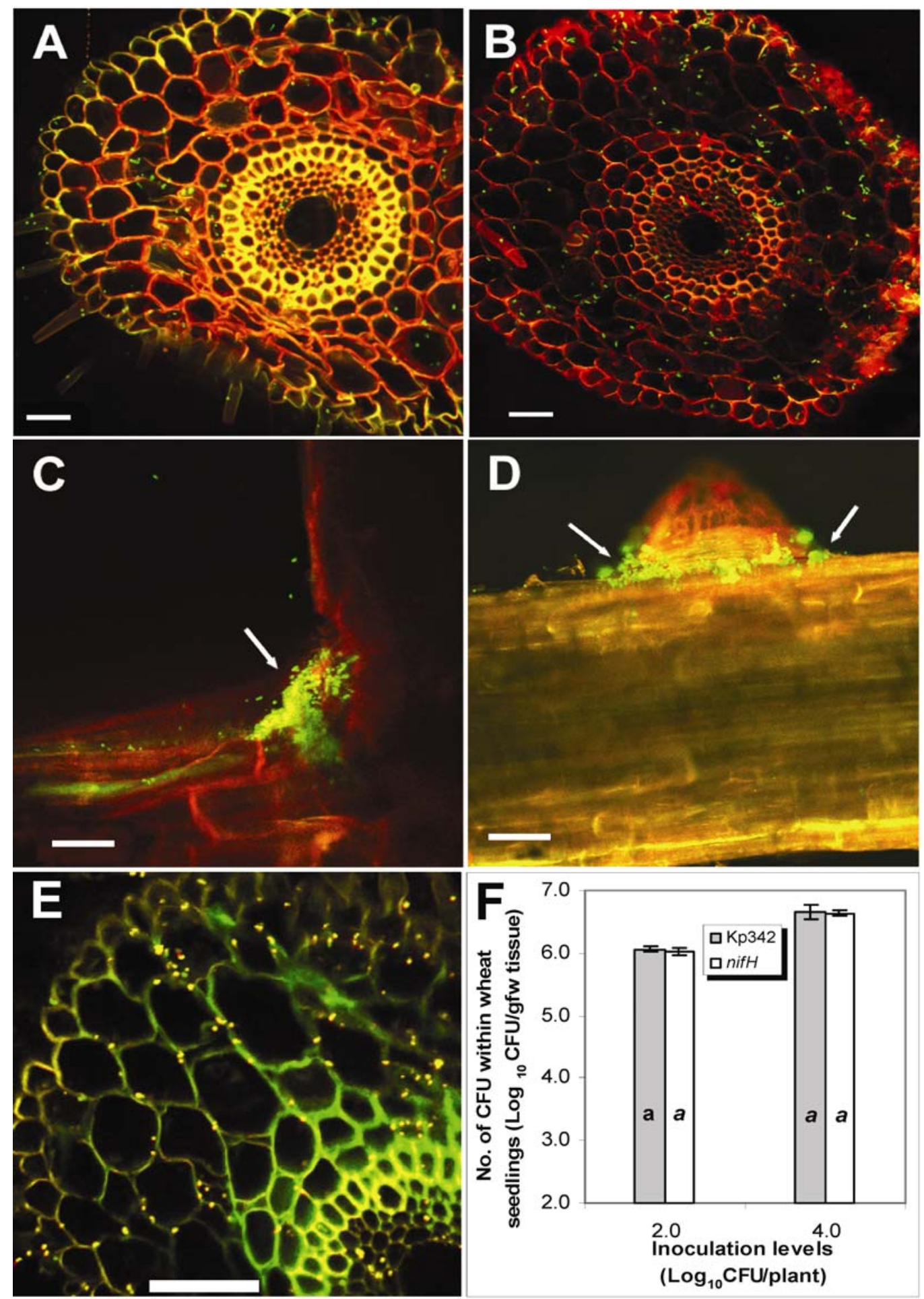

Fig. 4. A through D, Comparison of A and C, green fluorescent protein (GFP)-labeled (green) Klebsiella pneumoniae 342 (Kp342) wild-type and $\mathbf{B}$ and $\mathbf{D}$, GFP-labeled (green) Kp342 nifH mutant of spring wheat Triticum aestivum L. cv. Trenton root colonization. A and $\mathbf{B}$, Cross sections of spring wheat roots were examined as well as $\mathbf{C}$ and $\mathbf{D}$, lateral root emergence Bars $(50 \mu \mathrm{m})$. E, Immunolocalization of NifH produced by GFP-labeled Kp342 in root cross section. Cells are seen in yellow as the fluorophores of NifH (red) and GFP-labeled Kp342 are colocalized (yellow) Bars (50 um). F, Number of CFU recovered from the interior of roots T. aestivum L. cv. Trenton. Plants were inoculated with K. pneumoniae strain 342 (closed columns) and nifH mutant of Kp342 (open column) at $10^{2}$ and $10^{4} \mathrm{CFU} /$ plant inoculum level. The columns represent the means of each treatment. Each treatment consists of four replicates and each replicate consists of four plants. The bars represent the standard errors about the mean; gfw, gram (fresh weight). 
phytin mass with the Kp342 treatment compared with the nifH control was statistically significant at the $1 \%$ level of confidence in both plant growth media.

Kp342 was present within the roots of these plants and produced dinitrogenase reductase in planta (Fig. 4). The concentrations of Kp342 and nifH mutant cells in the roots were identical, regardless of the number of cells in the inoculum (Fig. 4F). Confocal images of root cross-sections and around lateral root emergence showed similar colonization patterns and abundance by both strains (Fig. 4A through E). Thus, the lack of nutritional benefit from the nifH cells was not caused by a failure of the mutant to colonize the exterior or interior of roots. Dinitrogenase reductase production by green fluorescent protein (GFP)-labeled Kp342 cells in roots was determined by scanning confocal laser microscopy (Fig. 4E). As done previously in maize (Chelius and Triplett 2000), the colocalization of both fluorophores (green for GFP and red for NifH) renders a yellow color, allowing the simultaneous localization of wild-type Kp342 expressing NifH. NifH expression by Kp342 was observed in several areas of the roots, including cross sections (Fig. 4E).

The $\mathrm{N}_{2}$ fixation phenotype described here is specific to $\mathrm{cv}$. Trenton. Nitrogen-deficiency symptoms were not relieved in cvs. Russ or Stoa with Kp342 inoculation but biomass did increase significantly in Stoa (Table 1).

\section{DISCUSSION}

Previous attempts to demonstrate nitrogen fixation in wheat have shown little if any fixed $\mathrm{N}$ provided by diazotrophic bacteria. Rennie and associates (1983) used ${ }^{15} \mathrm{~N}$ isotope dilution to show that up to $32 \%$ of the $\mathrm{N}$ in wheat plants of one cultivar was derived from the atmosphere following inoculation with strains of Bacillus polymyxa and Azospirillum brasilense, but there was no increase in $\mathrm{N}$ concentration in the plants compared with the uninoculated control and there was no report of increased plant growth or a relief of nitrogen-deficiency symptoms. Lethbridge and Davidson (1983) were unable to see $\mathrm{N}_{2}$ fixation in some of the same wheat lines using some of the same bacteria as inoculants. Boddey and associates (1986) also were unable to observe fixed $\mathrm{N}$ in wheat from inoculation with Azospirillum strains. Kucey (1988) observed small amounts of fixed $\mathrm{N}$ (up to $11 \%$ of plant $\mathrm{N}$ ) in field-grown wheat plants, but the authors suggested that might be in error because the ${ }^{15} \mathrm{~N}$ was not uniformly distributed with depth as it was in this work. In all of these cases, $\mathrm{Nif}^{-}$mutants never were used as controls. Bremer and associates (1995) reported that very little $\mathrm{N}_{2}$ was fixed in wheat plants cultured in the greenhouse. However, these plants were not inoculated with any diazotrophs.

In this work, the evidence of nitrogen fixation in wheat by Kp342 met all of the criteria for such experiments, as outlined

Table 1. Comparison of three wheat cultivars for their ability to enhance growth in the greenhouse and relieve nitrogen-deficiency symptoms upon inoculation with Klebsiella pneumoniae $342(\mathrm{Kp} 342)^{\mathrm{a}}$

\begin{tabular}{lllllc}
\hline & \multicolumn{2}{c}{$\begin{array}{c}\text { Dry weight } \\
\text { Cultivar }\end{array}$} & & & \multicolumn{2}{c}{$\begin{array}{c}\text { Chlorophyll } \\
\text { Kp342 }\end{array}$} & Khoots/plant) \pm s.e. & & Uninoc & & Kp342 & Uninoc \\
\cline { 2 - 3 } \cline { 5 - 6 } Russ & $416 \pm 3$ & $402 \pm 2$ & & $26.2 \pm 1.2$ & $25.3 \pm 1.4$ \\
Stoa & $380 \pm 4$ & $269 \pm 1$ & & $26.8 \pm 3.7$ & $26.1 \pm 0.5$ \\
Trenton & $790 \pm 13$ & $257 \pm 4$ & & $35.6 \pm 3.4$ & $22.9 \pm 3.9$ \\
\hline
\end{tabular}

${ }^{a}$ Measurements were taken after 6 weeks of growth in the absence of nitrogen fertilizer in a sand-vermiculite mixture. Biomass is measured as the dry weight of shoots per plant. Nitrogen deficiency measured by assaying the amount of chlorophyll in arbitrary units per unit area in the leaves. Kp342 = K. pneumoniae 342, Uninoc $=$ uninoculated, and s.e. $=$ standard error about the mean. above. Compared with the uninoculated and nifH mutantinoculated controls, Kp342 inoculation resulted in increased dry weight, chlorophyll content, total $\mathrm{N}$, and $\mathrm{N}$ concentration. In addition, the nitrogen-deficiency symptoms were relieved and ${ }^{15} \mathrm{~N}$ was diluted in the plant tissue and in chlorophyll as a result of Kp342 inoculation. Production of dinitrogenase reductase within the plant by Kp342 also is shown. Nearly all of these criteria were met in the inoculation of sugarcane with Gluconacetobacter diazotrophicus PAl5 (Sevilla et al. 2001). In these experiments, ${ }^{15} \mathrm{~N}_{2}$ was incorporated directly into the plants following inoculation with PAl5 but not following inoculation with an nifD mutant. However, the authors did not determine whether fixed $\mathrm{N}$ was incorporated into a plant product. Also, the $\mathrm{N}$ concentration in the plant tissue did not increase significantly and the authors did not determine nitrogenase expression by the bacteria in planta.

Small amounts of nitrogen fixation in Kallar grass upon inoculation with Azoarcus sp. BH72 may occur (Hurek et al. 2002). Although dry matter and total $\mathrm{N}$ increases in BH72inoculated plants were observed compared with the nifK mutant control, the nitrogen concentration in the plant actually decreased with $\mathrm{BH} 72$ inoculation. No evidence was presented to show that BH72 could relieve nitrogen-deficiency symptoms. A decline in ${ }^{15} \mathrm{~N}$ natural abundance was observed in BH72-inoculated plants compared with the controls, as expected if nitrogen fixation was occurring, but this was significant only in roots, not shoots. Natural abundance changes in ${ }^{15} \mathrm{~N}$ were not measured in any plant product and the authors were unable to confirm Koch's postulates because they failed to re-isolate $\mathrm{BH} 72$ after inoculation. Therefore, although fixed $\mathrm{N}$ may have been provided to Kallar grass by $\mathrm{BH} 72$, the amounts were just $1.4 \mathrm{mg}$ of $\mathrm{N} /$ plant for 2-month-old plants, an amount not sufficient to significantly improve the nutrition of the plant. The increases in total $\mathrm{N}$ in this work were 30 to $45 \mathrm{mg}$ of N/plant with 6-week-old plants.

Sevilla and associates (2001), Hurek and associates (2002), and this work all used different ${ }^{15} \mathrm{~N}$ assays to estimate the degree of nitrogen fixation in their respective systems. Each of these approaches has advantages and disadvantages (Boddey et al. 2000). The ${ }^{15} \mathrm{~N}_{2}$ reduction approach used by Sevilla and associates (2001) is the most direct approach for showing nitrogen fixation but it has three significant pitfalls. First, the plant-soil system must be in an airtight container, requiring replenishment of $\mathrm{CO}_{2}$ and $\mathrm{O}_{2}$ over time. Any leaks from that container will underestimate the amount of $\mathrm{N}_{2}$ fixation once ${ }^{15} \mathrm{~N}_{2}$ is added. Second, the ${ }^{15} \mathrm{~N}_{2}$ often is contaminated with ${ }^{15} \mathrm{NH}_{3}$. This can be avoided by passing the gas through a cold trap, but few studies using ${ }^{15} \mathrm{~N}_{2}$ describe their efforts to remove ammonia. Third, ${ }^{15} \mathrm{~N}_{2}$ reduction can only provide an estimate of $\mathrm{N}_{2}$ fixation during the short period of the assay. Other ${ }^{15} \mathrm{~N}$ approaches can give an estimate of $\mathrm{N}_{2}$ fixation throughout the growth period of the plant.

${ }^{15} \mathrm{~N}$ natural abundance assays used by Hurek and associates (2002) are most useful in field situations where ${ }^{15} \mathrm{~N}_{2}$ assays are not practical and where ${ }^{15} \mathrm{~N}$ isotope dilution assays are impossible because the soil cannot be uniformly labeled with ${ }^{15} \mathrm{~N}$. The problem with ${ }^{15} \mathrm{~N}$ natural abundance assays is that they are much less sensitive than ${ }^{15} \mathrm{~N}$ isotope dilution assays where the ${ }^{15} \mathrm{~N}$ isotope can be enriched. These measurements also require plants that are not fixing $\mathrm{N}_{2}$ in order to provide a baseline control to give confidence in the measurements.

The ${ }^{15} \mathrm{~N}$ isotope dilution approach used here requires that the ${ }^{15} \mathrm{~N}$ isotope be uniformly labeled in the soil prior to sowing the seed. In the experiments described here, the label was added to the soil mix and mixed thoroughly twice daily for 2 weeks prior to planting. Once the condition of mixing is satisfied, the ${ }^{15} \mathrm{~N}$ isotope dilution technique provides an excellent 
measure of $\mathrm{N}_{2}$ fixation by a plant-bacterial interaction provided that the appropriate non- $\mathrm{N}_{2}$-fixing controls are included, such as an uninoculated control and control where plants are inoculated with a Nif ${ }^{-}$mutant. According to Boddey and associates (2000), this approach has provided the most reliable estimates of nitrogen fixation in grasses to date because it gives an estimate of nitrogen fixation from germination to harvest. This approach was chosen for these experiments because the ${ }^{15} \mathrm{~N}$ label could be thoroughly mixed in the soil mixtures prior to planting under these growing conditions and because an estimate of nitrogen fixation throughout the growth period was desired. The use of ${ }^{15} \mathrm{~N}$ isotope dilution for demonstrating nitrogen fixation in grasses has been reported several times (Boddey et al. 1986; Miranda and Boddy 1987; Shrestha and Ladha 1996; Urquiaga et al. 1992; Wu et al. 1995).

The genotypic differences observed in the response to Kp342 provide a genetic tool for understanding the Kp342-Trenton wheat association. Kp342-inoculated Trenton wheat plants were not as large or as vigorous as wheat seedlings provided $10 \mathrm{mM}$ $\mathrm{KNO}_{3}$ in the nutrient solution. A genetic analysis of the genotypic differences will allow us to understand how Kp342 provides this benefit to this genotype and in no other genotype of tested to date, including Russ and Stoa wheat, Morex and Steptoe barley, Nipponbarre rice, and several lines of maize. Understanding this association should result in the improvement of the Kp342-Trenton association and in the extension of this phenotype to other cultivars of wheat and to other grasses.

\section{MATERIALS AND METHODS}

Kp342 (Chelius and Triplett 2000) and an nifH mutant of Kp342 were grown overnight on Luria-Bertani (LB) agar plates at $28^{\circ} \mathrm{C}$. DNA:DNA hybridization assays have classified Kp342 as a member of $K$. pneumoniae (Dong et al. 2003a). The four treatments in each experiment included uninoculated plants and plants inoculated with Kp342, the nifH mutant of $\mathrm{Kp} 342$, and dead cells of Kp342. Prior to inoculation, Kp342 and nifH mutant cells were resuspended in phosphate-buffered saline, creating a thick cell suspension containing $5 \times 10^{9}$ $\mathrm{CFU} / \mathrm{ml}$. For dead cells, Kp342 was cultured and resuspended as described above, but autoclaved for $30 \mathrm{~min}$. The heat-killed cell suspension was allowed to reach room temperature before it was applied to wheat seed. Cell death was confirmed by failure to grow on LB agar.

The nifH mutant was constructed as follows. Primers nifH1f (5'-GCCTGCAGATGACCATGCGTCAATGCGCC-3') and nifH876r (5'-GCGAATTCCGCGTTTTCTTCGGCGGCGGT$3^{\prime}$ ), based on the nifH sequence of $K$. oxytoca M5a1 (formerly K. pneumoniae M5a1) (Suárez et al. 1995), were used with 100 ng of Kp342 DNA in polymerase chain reaction (PCR) using the conditions described previously (Chelius and Triplett 2000). The PCR product was purified with a Qiagen PCR purification kit and then ligated to pGEM-T Easy vector. A 1.7-kb fragment containing nifH gene and part of nifD was excised from pSA30 by double digestion with EcoRI and BamHI. The nifHDKY operon from $K$. pneumoniae is present in pSA30 (Cannon et al. 1979). This fragment was inserted into EcoRI/BamHI-digested vector pUC18, resulting in plasmid $\mathrm{pH} 1$. A 1.4-kb fragment from pKRP11 (Reece and Phillips 1995) containing nptII downstream of a constitutive promoter was excised with HindIII and blunted with Klenow. Following BglII digestion of $\mathrm{pH} 1$ and subsequent blunting, pI1 was created by inserting the fragment from pKRP11 into the $B g l I I$ site of $\mathrm{pH} 1$. To exchange the inserted nifH for the wild-type allele on the chromosome, the 3.1$\mathrm{kb}$ fragment containing nifHD'-Km was excised from $\mathrm{pI} 1$ by digestion with EcoRI and PstI. This fragment was blunted and ligated into the PstI/SmaI-digested plasmid pJQ200KS+ fol- lowed by marker exchange (Scupham and Triplett 1997). Nif isolates then were selected on an $\mathrm{N}$-free medium with ampicillin and kanamycin. Marker exchange was confirmed by Southern hybridization with $n p t I I$ in isolates with no acetylene reduction activity.

The soil mixtures for each experiment were perlite and vermiculite, each mixed with sand in a 1:1 ratio by volume. Once mixed, the two soil mixtures were autoclaved at $121^{\circ} \mathrm{C}$ for $2 \mathrm{~h}$, allowed to cool overnight, and autoclaved again for $2 \mathrm{~h}$. The soil was allowed to cool to room temperature before adding 10 mg of ${ }^{15} \mathrm{NH}_{4} \mathrm{NO}_{3}$ (11.7 atom percent ${ }^{15} \mathrm{~N}$ excess) per kilogram wet weight of soil. To ensure the proper distribution of ${ }^{15} \mathrm{~N}$, the soil was mixed thoroughly twice daily for 2 weeks prior to planting. Finally, 2-liter pots were filled with approximately $2.5 \mathrm{~kg}$ of the ${ }^{15} \mathrm{~N}$-labeled soil mixture.

Seeds of Triticum aestivum L. cv. Trenton (a commercial cultivar) were surface sterilized as described previously (Chelius and Triplett 2000). After the surface sterilization, seed were submerged in the appropriate inoculum suspension described above at room temperature for approximately $2 \mathrm{~h}$ and then five seed per pot were placed in the soil mixtures. The remaining cell suspension was applied in equal amounts on top of the planted seed. After plants emerged, they were thinned to two plants per pot. There were 10 replicates per treatment. To measure chlorophyll, a Minolta SPAD 502 meter was used. Relative chlorophyll concentration is unitless and is a ratio of transmittance between red $(650 \mathrm{~nm})$ and infrared $(940 \mathrm{~nm})$ emissions through the leaf.

Plants were grown under greenhouse conditions, with 10-h nights at $21^{\circ} \mathrm{C}\left( \pm 2^{\circ} \mathrm{C}\right)$ and 14 -h days at $23^{\circ} \mathrm{C}\left( \pm 2^{\circ} \mathrm{C}\right)$. Artificial light ensured a minimum light level of $120 \mu$ einsteins $/ \mathrm{m}^{2} / \mathrm{s}$. Plants were watered as needed with a nutrient solution containing $5 \mu \mathrm{M} \mathrm{CaCl}, 1.25 \mu \mathrm{M} \mathrm{MgSO}_{4}, 5 \mu \mathrm{M} \mathrm{KCl}, 1 \mu \mathrm{M}$ $\mathrm{KH}_{2} \mathrm{PO}_{4}, 0.162 \mu \mathrm{M} \mathrm{FeSO}$, and micronutrients consisting of $2.91 \mathrm{nM} \mathrm{H}_{3} \mathrm{BO}_{3}, 1.14 \mathrm{nM} \mathrm{MnSO}, 0.76 \mathrm{nM} \mathrm{ZnSO}_{4}, 0.13 \mathrm{nM}$ $\mathrm{NaMoO}_{4}, 0.14 \mathrm{nM} \mathrm{NiCl}, 0.013 \mathrm{nM} \mathrm{CoCl}$, and $0.19 \mathrm{nM}$ $\mathrm{CuSO}_{4}$. Six weeks after planting, plants were washed to remove the attached soil mix. Roots and shoots were separated and dried at $65^{\circ} \mathrm{C}$ for $48 \mathrm{~h}$ and ground through a $0.5-\mathrm{mm}$ mesh. Roots and shoots $(10 \mathrm{mg})$ were assayed for ${ }^{15} \mathrm{~N}$ content by mass spectrometry. Using these data, the percent $\mathrm{N}$ in plant tissue derived from the atmosphere was estimated from ${ }^{15} \mathrm{~N}$ tissue analysis of roots and shoots. Chlorophyll ${ }^{15} \mathrm{~N}$ content was determined by mass spectrometry after acidification to pheophytin (Kahn et al. 2002).

Sand-perlite and sand-vermiculite subsamples (six of each) and seed were tested for total $\mathrm{N}$ content by Kjeldahl analysis. The extent of endophytic colonization, inoculum preparation, planting, in planta $\mathrm{NifH}$ visualization, statistics, and harvesting were done as described previously (Chelius and Triplett 2000; Dong et al. 2003b and c).

\section{ACKNOWLEDGMENTS}

We thank the University of Wisconsin Soil and Plant Analysis Laboratory, the University of California-Davis Stable Isotope Facility, and the University of Florida mass spectrometry facility in the Chemistry Department for the total $\mathrm{N}$ and ${ }^{15} \mathrm{~N}$ analyses described in this work. We thank the Consortium for Plant Biotechnology Research, Cargill, Inc., Agribiotics Inc., the College of Agricultural and Life Sciences of the University of WisconsinMadison, and the Florida Agricultural Experiment Station for their financial support of this work. This work is approved for publication as Journal Series No. R-10354 of the Florida Agruicultural Experiment Station.

\section{LITERATURE CITED}

Boddey, R. M., Baldani, V. L. D., Baldani, J. I., and Döbereiner J, 1986. Effect of inoculation of Azospirillum spp. on nitrogen accumulation by field-grown wheat. Plant Soil 95:109-121. 
Boddey, R. M., and Chalk, P. M. 1983. The ${ }^{15} \mathrm{~N}$-isotope dilution technique applied to the estimation of biological nitrogen fixation associated with Paspalum notatum cv. Batatais in the field. Soil. Biol. Biochem. 15:2532.

Bremer, E., Janzen, H. H., and Gilbertson, C. 1995. Evidence against associative $\mathrm{N}_{2}$ fixation as a significant $\mathrm{N}$ source in long-term wheat plots. Plant Soil 175:13-19.

Cannon, F. C., Riedel, G. E., and Ausubel, F. M. 1979. Overlapping sequences of $K$. pneumoniae nif DNA cloned and characterized. Mol. Gen. Genet. 174:59-66.

Chelius, M. K., and Triplett, E. W. 2000. Immunolocalization of dinitrogenase reductase produced by Klebsiella pneumoniae in association with Zea mays L. Appl. Environ. Microbiol. 66:783-787.

Dixon, R., Cheng, Q., Shen, G.-F., Day, A., and Dowson-Day, M. 1997. Nif gene transfer and expression in chloroplasts: Prospects and problems. Plant Soil 194:193-203.

Dong Y., Chelius, M. K., Brisse, S., Kozyrovska, N., Kovtunovych, G., Podschun, R., and Triplett, E. W. 2003a. Comparisons between two Klebsiella: the plant endophyte Kp342 and a clinical isolate, K. pneumoniae MGH78578. Symbiosis 35:247-259.

Dong, Y., Iniguez, A. L., Ahmer, B. M. M., and Triplett, E. W. 2003b. Kinetics and strain specificity of rhizosphere and endophytic colonization by enteric bacteria on seedlings of Medicago sativa and Medicago truncatula. Appl. Environ. Microbiol. 69:1783-1790.

Dong, Y., Iniguez, A. L., and Triplett, E. W. 2003c. Quantitative assessments of the host range and strain specificity of endophytic colonization by Klebsiella pneumoniae 342. Plant Soil 257:49-59.

Hurek, T., Handley, L. L., Reinhold-Hurek, B., and Piche, Y. 2002. Azoarcus grass endophytes contribute fixed nitrogen to the plant in an unculturable state. Mol. Plant-Microbe Interact. 15:233-242.

Kahn, M. L., Parra-Colmenares, A., Ford, C. L., Kaser, F., McCaskill, D., and Ketchum, R. E. 2002. A mass spectrometry method for measuring ${ }^{15} \mathrm{~N}$ incorporation into pheophytin. Anal. Biochem. 307:219-225.

Kucey, R. M. N. 1988. Alteration of size of wheat root systems and nitrogen fixation by associative nitrogen-fixing bacteria under field conditions. Can. J. Microbiol. 34:735-739.
Lethbridge, G., and Davidson, M. S. 1983. Root-associated nitrogen-fixing bacteria and their role in the nitrogen nutrition of wheat estimated by ${ }^{15} \mathrm{~N}$ isotope-dilution. Soil Biol. Biochem. 15:365-374.

Miranda, C. H. B., and Boddey, R. M. 1987. Estimation of biological nitrogen fixation associated with 11 ecotypes of Panicum maximum grown in nitrogen-15-labeled soil. Agron. J. 79:558-563.

Reece, K. S., and Phillips, G. J. 1995. New plasmids carrying antibioticresistance cassettes. Gene 165:141-142.

Rennie, R. J., deFreitas, J. R., Ruschel, A. P., and Vose, P. V. $1983 .{ }^{15} \mathrm{~N}$ isotope dilution to quantify dinitrogen $\left(\mathrm{N}_{2}\right)$ fixation associated with $\mathrm{Ca}$ nadian and Brazilian wheat. Can. J. Bot. 61:1667-1671.

Riggs, P. J., Chelius, M. K., Iniguez, A. L., Kaeppler, S. M., and Triplett, E. W. 2001. Enhanced maize productivity by inoculation with diazotrophic bacteria. Aust. J. Plant Physiol. 28:829-836.

Scupham, A. J., and Triplett, E. W. 1997. Isolation and characterization of the UDP-glucose 4'-epimerase gene, galE, from Brucella abortus S2308. Gene 202:53-59

Sevilla, M., Burris, R. H., Gunapala, N., and Kennedy, C. 2001. Comparison of benefit to sugarcane plant growth and ${ }^{15} \mathrm{~N}_{2}$ incorporation following inoculation of sterile plants with Acetobacter diazotrophicus wild-type and $\mathrm{Nif}^{-}$mutant strains. Mol. Plant-Microbe Interact. 14:358-366.

Shrestha, R. K., and Ladha, J. K. 1996. Genotypic variation in promotion of rice dinitrogen fixation as determined by nitrogen-15 dilution. Soil Sci. Soc. Am. J. 60:1815-1821.

Suárez, M., Ferrer, E., Garrido-Pertierra, A., and Martín, M. 1995. Purification and characterization of the 3-hydroxybenzoate-6-hydroxylase from Klebsiella pneumoniae. FEMS (Fed. Eur. Microbiol. Soc.) Microbiol. Lett. 126:283-290.

Urquiaga, S., Cruz, K. H. S., and Boddey, R. M. 1992. Contribution of nitrogen fixation to sugar cane-Nitrogen-15 and nitrogen-balance estimates. Soil Sci. Soc. Am. J. 56:105-114.

Wu, P., Zhang, G., Ladha, J. K., McCouch, S. R., and Huang, N. 1995. Molecular-marker-facilitated investigation on the ability to stimulate $\mathrm{N}_{2}$ fixation in the rhizosphere by irrigated rice plants. Theor. Appl. Genetic. 91:1177-1183. 